\section{Cahiers de Narratologie}

Analyse et théorie narratives

$16 \mid 2009$

Images et récits

\title{
Le récit du temps photographié
}

Józef Bury

\section{OpenEdition}

Journals

Édition électronique

URL : http://journals.openedition.org/narratologie/1022

DOI : 10.4000/narratologie.1022

ISSN : 1765-307X

Éditeur

LIRCES

\section{Référence électronique}

Józef Bury, «Le récit du temps photographié », Cahiers de Narratologie [En ligne], 16 | 2009, mis en ligne le 29 novembre 2010, consulté le 21 décembre 2020. URL : http://journals.openedition.org/ narratologie/1022 ; DOI : https://doi.org/10.4000/narratologie.1022

Ce document a été généré automatiquement le 21 décembre 2020.

\section{(c) (i) (9)}

Cahiers de Narratologie - Analyse et théorie narratives est mis à disposition selon les termes de la licence Creative Commons Attribution - Pas d'Utilisation Commerciale - Pas de Modification 4.0 International. 


\title{
Le récit du temps photographié
}

\author{
Józef Bury
}

L'activité de récit consiste à construire des ensembles

temporels cohérents :

à configurer le temps

Paul Ricœur

1 La photographie est affaire de temps et toute photographie est une chronophotographie. Ces affirmations reflètent la conviction que j'ai acquise durant une longue période de l'expérimentation avec le médium photographique.

2 L'« ouverture » de la perspective temporelle de la photographie, et plus précisément, le dérèglement $\mathrm{du}$ paramètre temps, m'ont en particulier fait prendre conscience de l'importance de la temporalité, et m'ont conduit à restreindre la portée de l'aspect optique de la photographie. L'ensemble des paramètres optiques de la situation photographique - la mise au point, la distance de la focale (le grossissement macro et le rapprochement télé-objectif), l'ouverture du diaphragme et la profondeur de champ ne caractérisent que partiellement la photographie. Ils se laissent, pour ainsi dire, " réduire » voire éliminer de l'expérience photographique sans affecter la spécificité de cette dernière. Or, le temps apparaît comme un paramètre constant et spécifique de la photographie. Il est décisif dans toutes les configurations de l'appareil et dans toutes les situations de l'inscription photo-graphique, et cela dès la formation de l'image latente jusqu'à la lecture du résultat, en passant par sa "révélation » (argentique ou numérique). La temporalité de la photographie se laisse lire tout d'abord dans la photosensibilité qui n'est rien d'autre que la rapidité de réaction à la lumière. C'est la photosensibilité qui détermine la durée d'exposition à la lumière - le temps de pose. La photographie sans optique (par exemple par contact) rencontrera toujours le problème du temps, puisque la matière photosensible réagit dans le temps. C'est également la photosensibilité qui détermine, encore aujourd'hui, à l'heure des capteurs numériques, le contraste et le rendu des détails. Viennent ensuite le temps de développement (le temps de l'action chimique sur les sels d'argent affectés par la lumière) et la limitation temporelle $\mathrm{du}$ fixateur qui permet de rendre le dépôt lumineux résistant à toute altération, donc de limiter le temps d'exposition et finalement d'arrêter le processus 
photosensible. La fixation (la « désensibilisation ») de l'image est donc une intervention dans le temps de processus de la réaction photosensible. C'est une manipulation du temps de l'action de la lumière. Historiquement, c'est l'élaboration du fixateur qui marque le seuil de l'invention de la photographie.

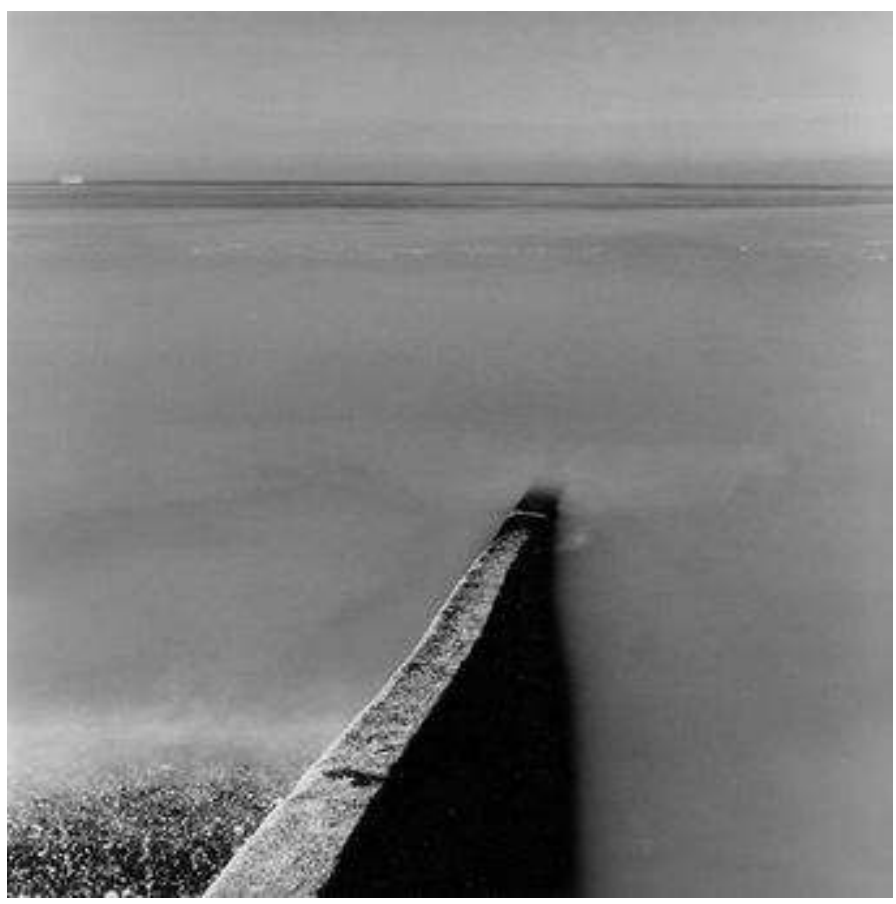

Józef Bury, Anamnèse 1.3. 1995. Photographie noir et blanc 47x47 cm

3 Le temps d'exposition de la plaque photosensible, ou du capteur numérique, (le temps de pose), quand il est situé au-delà de la durée nécessaire à la capture d'images conformes aux habitudes perceptives, décide du degré de révélation des régimes de mobilité du réel opérant en dehors de notre capacité du discernement visuel. Les résultats de la chronophotographie étant peu prévisibles, la stratégie temporelle met donc en doute la réalité perçue. En dehors de l'aspect iconographique des résultats, la chronophotographie pourrait alors être envisagée comme un moyen d'interroger le réel et sa capacité de se révéler à travers le médium photographique. Elle s'érige en un mode d' "expérience du réel à l'aide d'undispositif technique ", capable de soulever un questionnement lié aux possibilités de perception sensible, de la cognition et de la mémorisation de la réalité changeante. Elle permet donc de questionner les processus de constitution du savoir sur l'objet photographié. Privilégier l'aspect temporel, envisager la "chronophotographie", fait donc basculer la photographie du côté de l'expérience. 


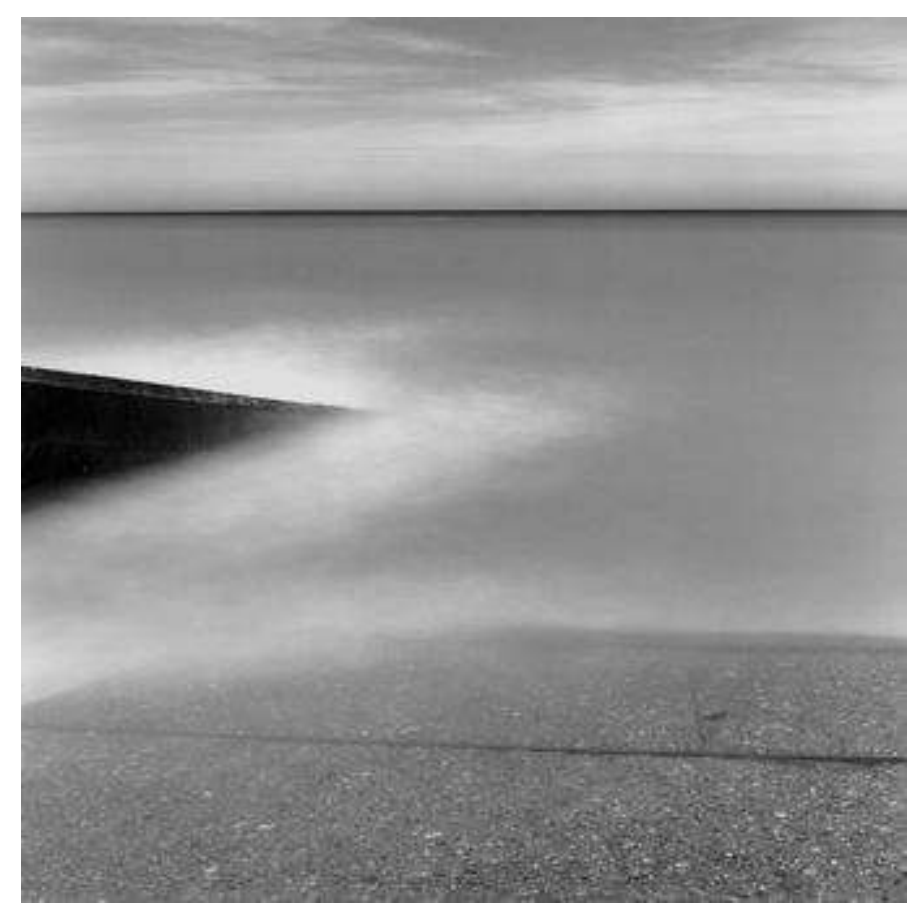

Józef Bury, Anamnèse 2.5. 1997. Photographie noir et blanc $47 \times 47 \mathrm{~cm}$

4 Par son caractère imprévisible, l'expérience chronophotographique conjugue plusieurs éléments déterminants. Tout d'abord, l'acte de photographier - l'utilisation du dispositif technique pour capter l'image du réel. Ensuite, pour le photographe, le fait d'être à côté d'un appareil qui enregistre dans le temps implique un acte de perception du réel dans la durée. En effet, l'appareil est hors de toute possibilité de contrôle par la perception sensible. La perception et le processus de mémorisation de l'expérience sont engagés dans leur épaisseur temporelle. Parallèlement, le résultat de la perception se trouve altéré par l'anticipation des résultats chronophotographiques. Enfin, les résultats photographiques sollicitent un processus cognitif (lecture des images chronophotographiques) afin de les reconnaître comme images signifiantes. En effet, les clichés obtenus dans ce type d'expérience ne sont que des résidus fragmentaires de l'expérience complète : photographique, perceptive et cognitive. Ils constituent la base d'enregistrement et permettent le retour de la mémoire de l'expérience. Ils servent donc de support à l'anamnèse photographique.

En effet, l'image photographique offre un récit, celui de la mémoire du temps de l'interaction de la matière photosensible avec la réalité changeante. Mais la différence (et la possibilité de comparaison) entre l'enregistrement chronophotographique et l'enregistrement d'un vécu de la même expérience dans la mémoire ouvre une autre possibilité : la possibilité de confrontation de deux modalités d'inscriptions du réel. « La conscience de ce fait me force à suspendre, en quelque sorte, la réalité perçue, puisque je sais qu'il y en a en même temps une autre, pour un autre dispositif, qui mémorise également le réel et ensuite donne des résultats différents des miens. [...] Je suis donc obligé d'admettre, pour un moment, l'écartement de ma propre connaissance du monde ${ }^{1} . »$ 


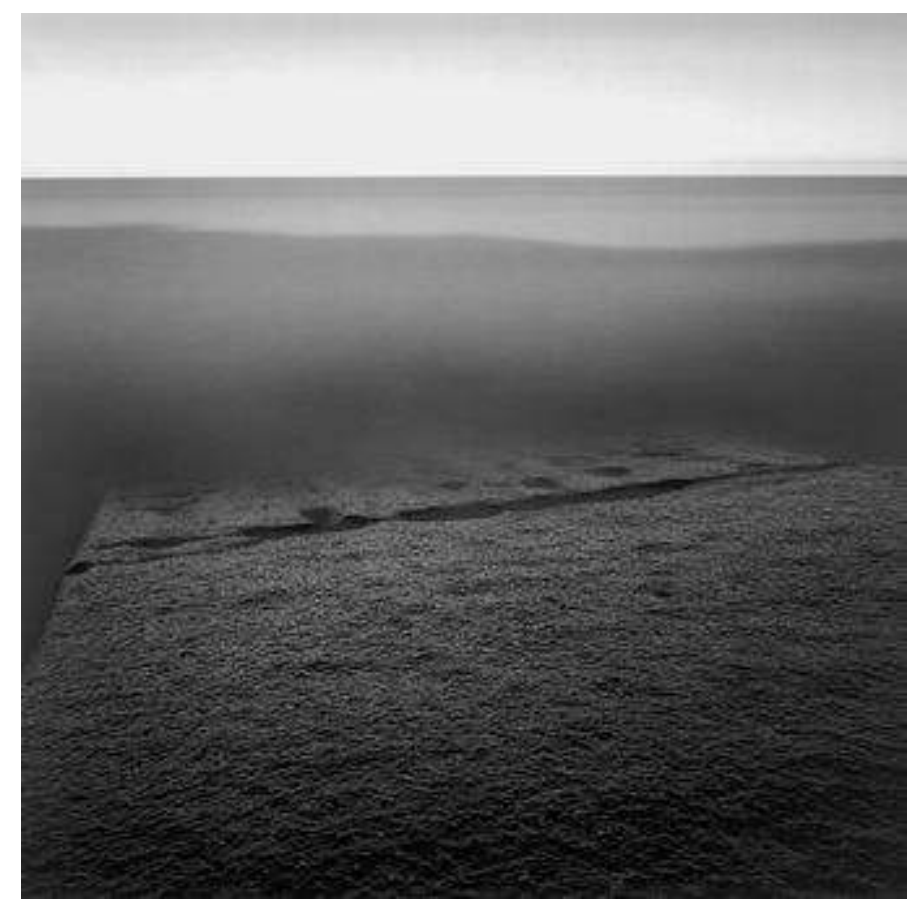

Józef Bury, Anamnèse 3.10. 1997. Photographie noir et blanc $47 \times 47 \mathrm{~cm}$

6 Contrairement à la prise de vue instantanée, la chronophotographie, qui restitue le réel étendu dans sa durée, implique une double difficulté interprétative ${ }^{2}$. D'abord, parce qu'elle renvoie à une réalité inaccessible à travers un acte perceptif habituel. Ensuite, parce que, gardant une structure résiduelle de son référent (et tout simplement par sa nature indicielle «malgré tout»), elle « exige» d'être "reconnue» comme image de «quelque chose ». La lecture de ces images requiert donc l'exercice de deux modalités intentionnelles conjointes: l'intentionnalité perceptive référentielle, d'une part; et d'autre part la validation des données faisant appel à la mémoire de l'expérience vécue. 


\section{ANNEXES}

Józef Bury, Mono-chrone 1.1. 2001. Photographie noir et blanc $47 \times 47 \mathrm{~cm}$

1.

\section{Autoportrait de Józef Bury}

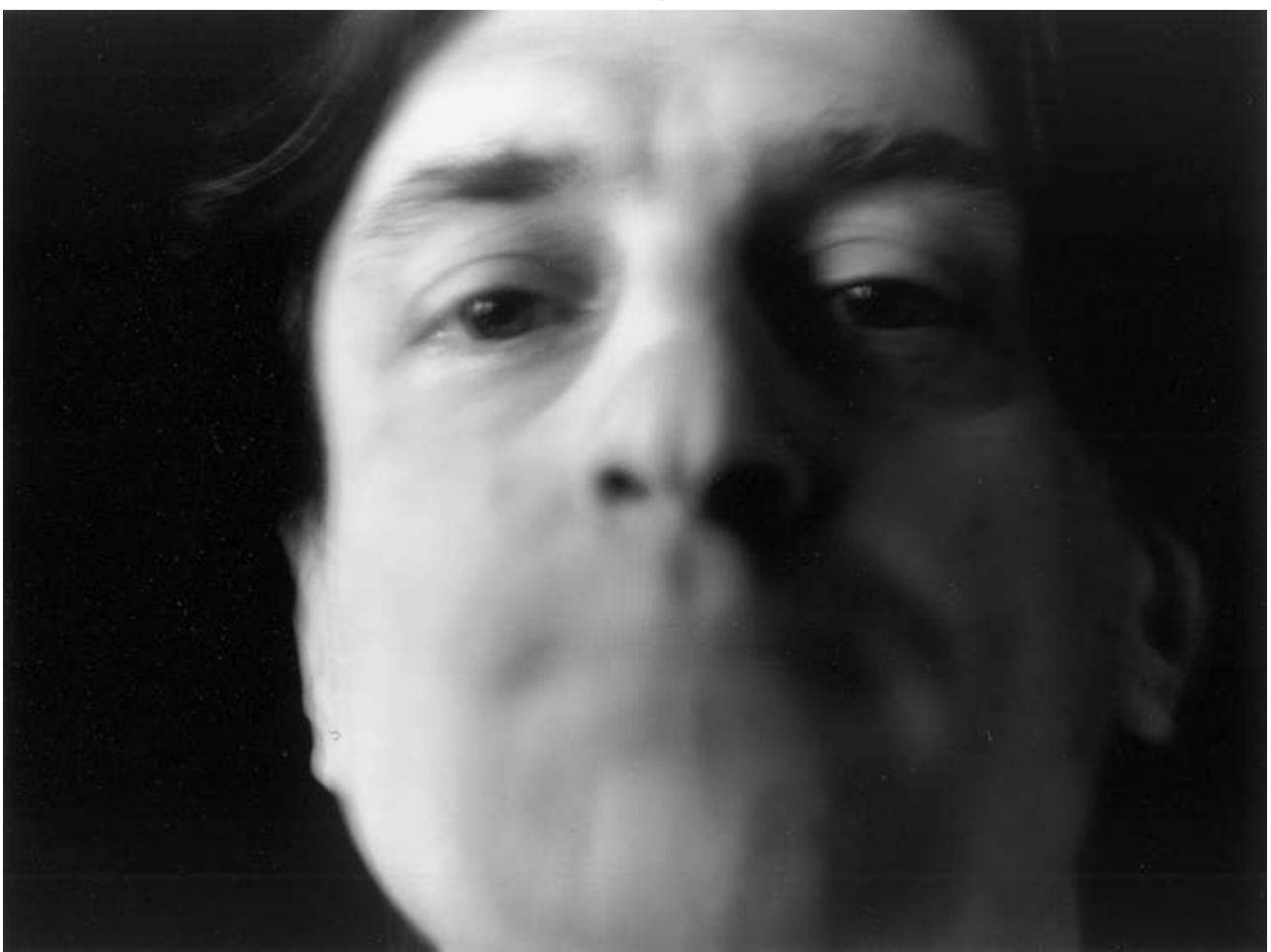

Józef Bury, Aggloportrait 7/4, 1999. Photographie noir et blanc 26x36cm.

\section{NOTES}

1. Voir également à ce sujet: Marcin Sobieszczanski, Les artistes et la perception - Entretiens avec Zbigniew Dlubak, Józef Bury, Ernesto Riveiro et Bernard Caillaud. Paris : L'Harmattan, coll. «L'art en bref ", $2000 . \quad$ http://books.google.fr/books? vid=ISBN2738496091\&id=4PUMCJpd4lUC\&dq=ISBN+2738496091\&ie=ISO-8859-1\&output=html 2. Concernant la lecture des images chronophotographiques, voir également : Józef Bury, David Piotrowski, «À propos de l'expérience chronophotographique », pp. 13-17, in : Bury, J. (dir.), Art subcognitif. 5e Rencontres Internationales d'Art de Katowice / Actes du colloque, Katowice: Contemporary Art Gallery, BWA Katowice 2005. http://art.action.anamnese.free.fr/chrono1.html 
INDEX

Mots-clés : Chronophotographie, photographie, temps, récit

\section{AUTEUR}

\section{JÓZEF BURY}

Józef Bury est un artiste transdisciplinaire, commissaire d'expositions et auteur de nombreux articles, conférences et traductions dans le domaine de l'art contemporain.

Il enseigne l'esthétique à l'École supérieure d'arts de Brest et dirige International Art Meeting Katowice. Mail : jozefbury@free.fr Web : http://jozefbury.free.fr/index.html 